\title{
PROPRIÉTÉS PHOTOCONDUCTRICES DES COUCHES MINCES DE SÉLÉNIUM AMORPHE
}

\author{
C. VIGER, C. VAUTIER et J. GRENET \\ Laboratoire de Physique des Couches minces, Faculté des Sciences et des Techniques, \\ 76130 Mont-Saint-Aignan, France
}

\begin{abstract}
Résumé. - L'étude systématique, en fonction de différents paramètres (longueur d'onde, intensité lumineuse, champ électrique et température), de la photoconduction dans les couches minces de sélénium amorphe nous a permis de montrer que les films sont composés en majeure partie d'anneaux $\mathrm{Se}_{8}$, qu'aux basses températures les états qui gouvernent la mobilité commandent la photoconduction. A $T=290 \mathrm{~K}$ et pour les champs élevés la photoconduction est assistée par le champ. Enfin l'application des théories de Davis [15] nous a permis de déterminer la largeur de la bande interdite, $E_{\mathrm{g}}=2,238 \mathrm{eV}$.
\end{abstract}

Abstract. - The variation of photoconductivity with various parameters (wavelength, light intensity, electric field and temperature) was studied on amorphous selenium thin films. The experimental results are enable us to suggest that the films are predominantly composed of $\mathrm{Se}_{8}$ rings. At low temperatures the photoconduction is controlled by the hopping in the mobility edge, whereas at room temperature the photogeneration is field-assisted. Lastly, the comparison between our experimental data and Davis' theories [15] permits us to calculate the band gap $E_{\mathrm{g}}=2.238 \mathrm{eV}$.

1. Introduction. - Parmi les phénomènes de transport dans les semiconducteurs amorphes la photoconduction est l'un des moins étudiés. Le sélénium amorphe se prête particulièrement bien à une telle étude car le photocourant $I_{\mathrm{ph}}$ est grand devant le courant d'obscurité $I_{\mathrm{d}}$ même pour de faibles éclairements $\left(I_{\mathrm{ph}} / I_{\mathrm{d}} \simeq 10^{3}\right.$ pour $\left.F=1 \mu \mathrm{W} / \mathrm{cm}^{2}\right)$.

L'étude que nous avons entreprise a été menée en faisant varier d'une manière systématique les paramètres pouvant influencer la photoconduction.

2. Méthodes expérimentales. - Les mesures décrites ici ont été effectuées in situ sur des couches d'épaisseurs comprises entre 4000 et $6000 \AA$. Ces couches sont obtenues par évaporation dans un vide de $10^{-8}$ torr. Lors de l'évaporation la température du substrat est maintenue à $290 \mathrm{~K}$ et la température dans le creuset est d'environ $470 \mathrm{~K}$.

L'éclairement des couches est obtenu par l'intermédiaire d'un monochromateur et d'une lampe quartzhalogène dont le flux est stabilisé à $10^{-4}$ près (la relaxation du photocourant s'étendant parfois sur plusieurs heures). Dans tout le domaine spectral étudié $(0,3$ à $0,52 \mu \mathrm{m}$ ) le rayonnement monochromatique est entièrement absorbé par la couche.

Sauf pour les mesures décrites au paragraphe 4 , le champ appliqué à la couche est $E=10^{7} \mathrm{~V} \cdot \mathrm{m}^{-1}$.

3. Réponse spectrale. - Sur la figure 1 nous avons représenté (courbe en traits pleins) la variation du photocourant - par photon absorbé - en fonction de la longueur d'onde. Sur la même figure nous avons également représenté les spectres de photoréponse du sélénium monoclinique et du sélénium hexagonal [1]. L'interprétation de ces spectres a été faite en tenant REVUE de PHYSIQUe APPLIQUéE. - T. 12, No 5, MAI 1977

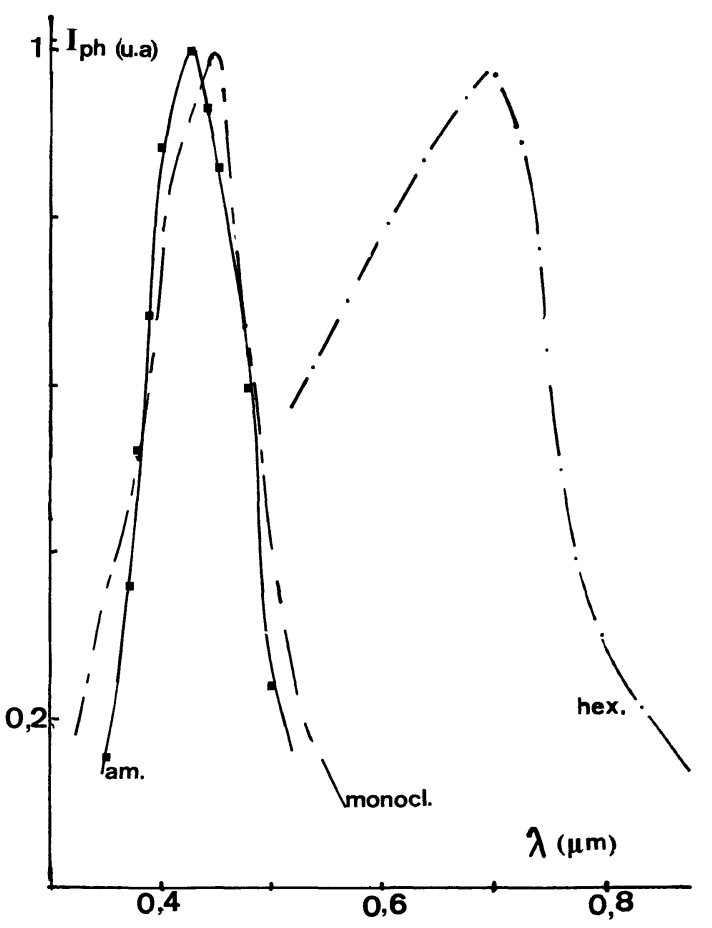

Fig. 1. - Spectres de photoréponse du sélénium amorphe et du sélénium cristallin monoclinique et hexagonal.

compte de la structure (anneaux ou chaînes) de ces deux types de sélénium cristallin. On remarque que le spectre du sélénium amorphe est très voisin du sélénium monoclinique.

Juskas et al. [2] ont montré que la variation de la conductibilité et de la mobilité des trous avec la température, lorsqu'on accomplit un cycle chauffage-refroidissement juste au-dessous de la température $T_{\mathrm{g}}$ pré- 
sentait une hystérésis que l'on retrouve dans le sélénium monoclinique. L'étude de l'influence des hautes pressions sur les processus électroniques [3] montre que les transitions observées dans les films de Se et dans le sélénium monoclinique sont très voisines.

Luckovsky [4] a montré qu'il y avait une similitude entre les spectres I. R. et Raman des deux espèces, mais il pense [5] que cette identité n'est pas une preuve irréfutable de la présence d'anneaux dans Se amorphe, ce que réfute les récents travaux de Zirke et Tausend [6].

L'ensemble de tous ces résultats, nous a conduit, en accord avec plusieurs auteurs $[3,6,7]$ à émettre l'hypothèse [8] que les couches minces de sélénium amorphe déposées sur des substrats à température ambiante sont formées en majeure partie par des anneaux $\mathrm{Se}_{8}$. Zirke [6] a montré de plus que ces anneaux étaient très certainement déformés confirmant ainsi les travaux de Kaplow et al. [9] sur les fonctions de distribution radiale.

4. Influence du champ électrique. - L'étude a été menée à $290 \mathrm{~K}$ pour plusieurs longueurs d'ondes et sur des couches de différentes épaisseurs (de 4000 à $6000 \AA$ ). Pour des champs supérieurs à

$$
2,8 \times 10^{6} \mathrm{~V} \cdot \mathrm{m}^{-1}
$$

le photocurant suit une loi linéaire en $E^{1 / 2}$ (Fig. 2). Il est

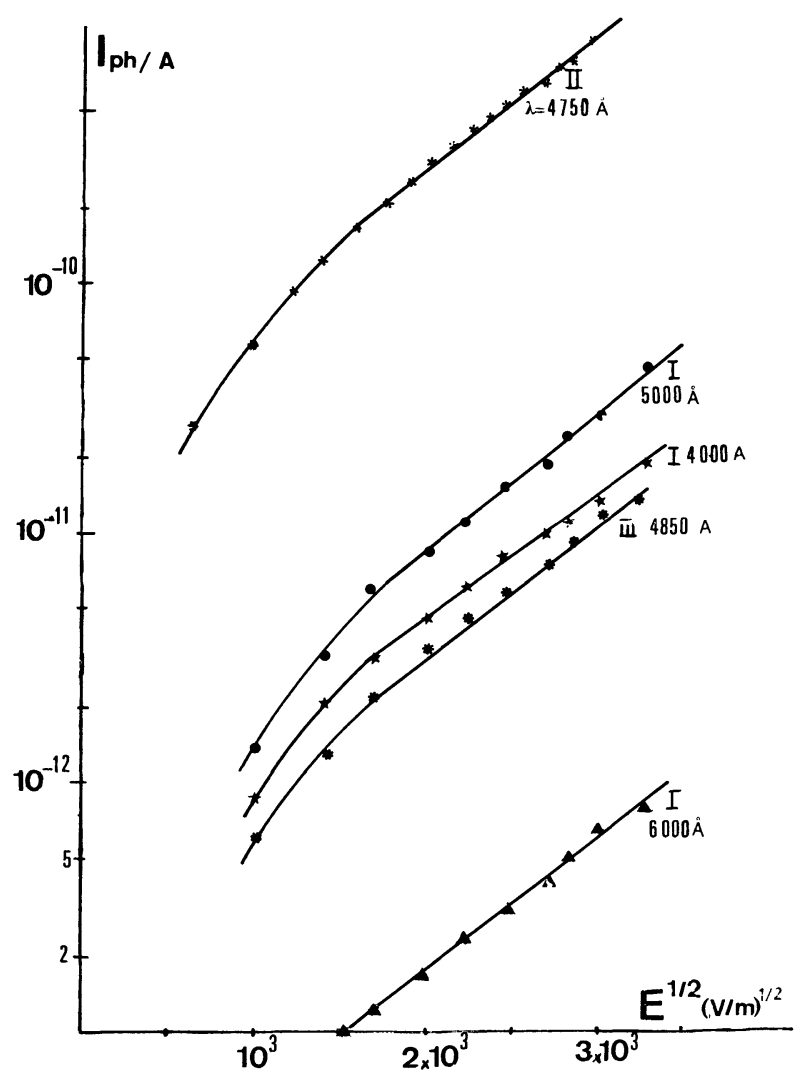

Fig. 2. - Variation du photocourant avec $E^{1 / 2}$ pour différentes longueurs d'onde et différentes couches (I. $e=6000 \AA$; II. $e=6000 \AA$; III. $e=4000 \AA$ ). classique de représenter une telle variation par une loi de Poole-Frenkel de la forme :

$$
I_{\mathrm{ph}}=B \exp \left[\frac{\beta_{\mathrm{PF}} E^{1 / 2}}{k T}-\frac{E_{0}}{k T}\right]
$$

dans laquelle la constante de Poole-Frenkel

$$
\beta_{\mathrm{PF}}=\left(\frac{e^{3}}{\pi \varepsilon}\right)^{1 / 2}
$$

vaut $4,589 \times 10^{-24} \mathrm{JV}^{-1 / 2} \mathrm{~m}^{1 / 2}$.

Les valeurs expérimentales déterminées à partir de la pente des courbes figurent dans le tableau ci-dessous.

$\begin{array}{ccc}\text { Couche } \mathrm{n}^{0} & \begin{array}{c}\text { Longueur } \\ \text { d'onde } \\ (\AA)\end{array} & \begin{array}{c}\beta_{\mathrm{PF}} \exp \times 10^{24} \\ \mathrm{JV}^{-1 / 2} \mathrm{~m}^{1 / 2}\end{array} \\ - & - & - \\ \text { I } & 4000 & 4,43 \\ & 5000 & 5,30 \\ \text { II } & 6000 & 5,26 \\ \text { III } & 4750 & 4,67 \\ & 4850 & 4,68\end{array}$

On remarque que les valeurs expérimentales sont très voisines de la valeur théorique et pratiquement indépendantes de la longueur d'onde, en contradiction avec les résultats obtenus par Pai et Ing [10].

5. Influence de la température. - La réponse spectrale pour diverses températures est représentée sur la figure $3:$ quelle que soit la température, la courbe présente un maximum pour $h v=3 \mathrm{eV}$; au-dessus de

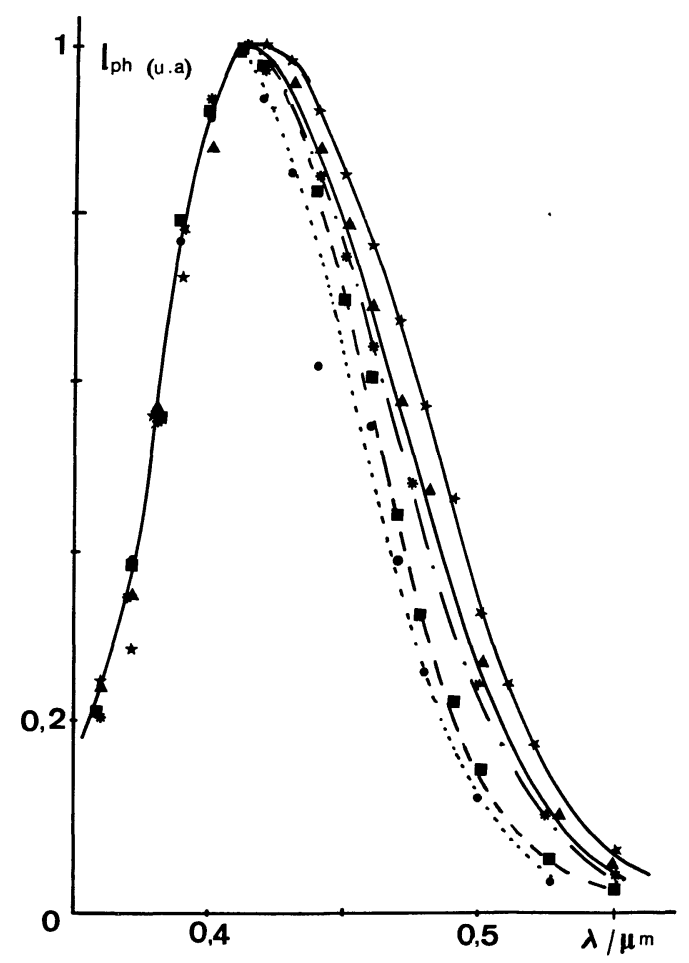

FIG. 3. - Réponse spectrale pour différentes températures $\star T=290 \mathrm{~K} ; \Delta T=266 \mathrm{~K} ; * T=248 \mathrm{~K} ; \square T=209 \mathrm{~K}$; - $T=182 \mathrm{~K}$. 
cette énergie la forme de la courbe est indépendante de la température alors qu'au-dessous la pente des courbes croît lorsque la température diminue. Ce comportement peut être attribué à une variation corrélative du coefficient d'absorption [11].

Dans tout ce paragraphe, nous considérons deux domaines d'éclairement, celui des forts éclairements pour lesquels le photocourant varie entre $10^{2}$ et $10^{4}$ fois le courant d'obscurité, et le domaine des faibles éclairements pour lesquels le photocourant est inférieur à 100 fois $I_{\mathrm{d}}$.

La figure 4 représente le comportement typique des courbes $I_{\mathrm{ph}}=f(F)$ pour les longueurs d'ondes absor-

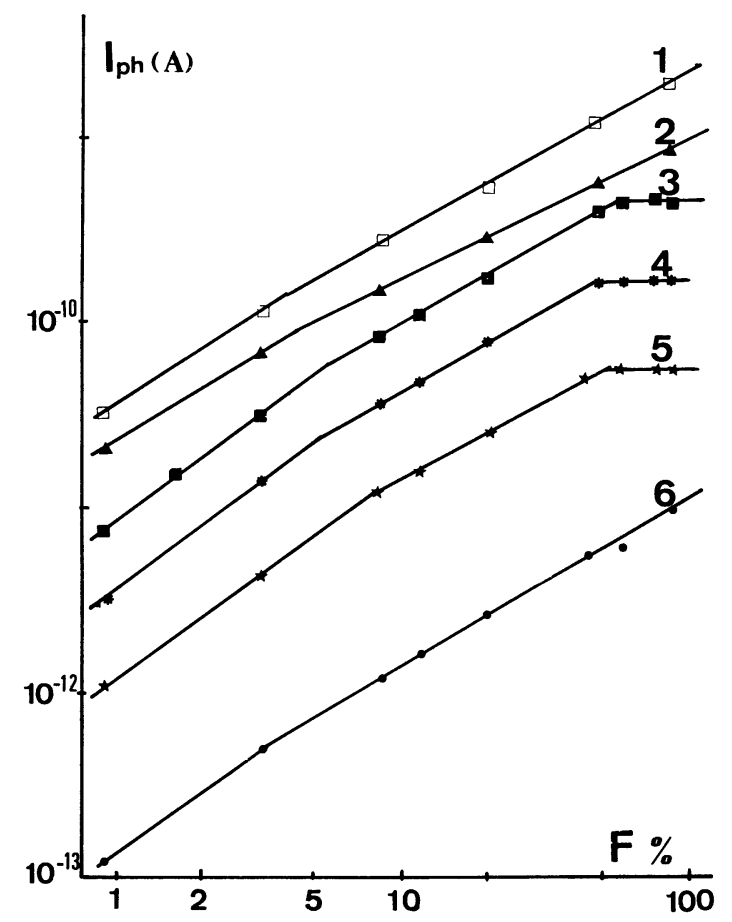

FIG. 4. - Influence de l'intensité lumineuse pour différentes températures : 1) $T=290 \mathrm{~K}$; 2) $T=268 \mathrm{~K}$; 3) $T=248 \mathrm{~K}$; 4) $T=224 \mathrm{~K}$; 5) $T=190 \mathrm{~K}$; 6) $T=155 \mathrm{~K}\left(E=10^{7} \mathrm{~V} / \mathrm{m}\right.$, $\lambda=0,45 \mu \mathrm{m})$.

bées dans le domaine des forts éclairements. Sauf pour les températures comprises entre 190 et $248 \mathrm{~K}$ pour lesquelles une saturation apparaît, toutes les courbes présentent deux pentes dont les valeurs respectives sont 1 (pour des faibles valeurs de $F$ ) et 0,86 ; ces valeurs sont indépendantes de la longueur d'onde. Par contre dans le domaine des faibles éclairements les courbes ne présentent plus qu'une seule pente, c'est-à-dire que la variation est de la forme

$$
I_{\mathrm{ph}}=k F^{n} \text {. }
$$

La variation de $\mathrm{n}$ avec la température est représentée sur la figure 5 .

Sur la figure 6, nous avons représenté la variation du photocourant en fonction de la température pour les deux domaines étudiés.

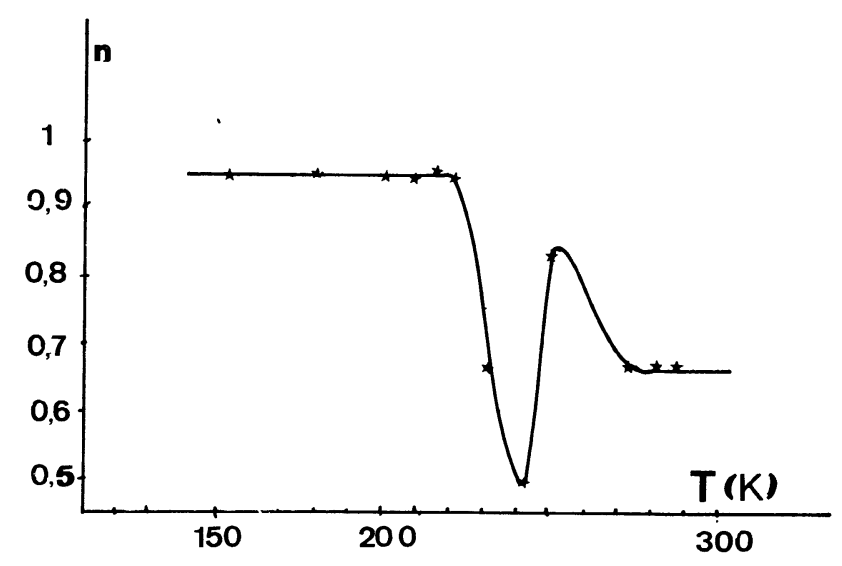

Fig. 5. - Variation de $n$ avec la température $\left(I_{\mathrm{ph}}=k F^{n}\right)$.

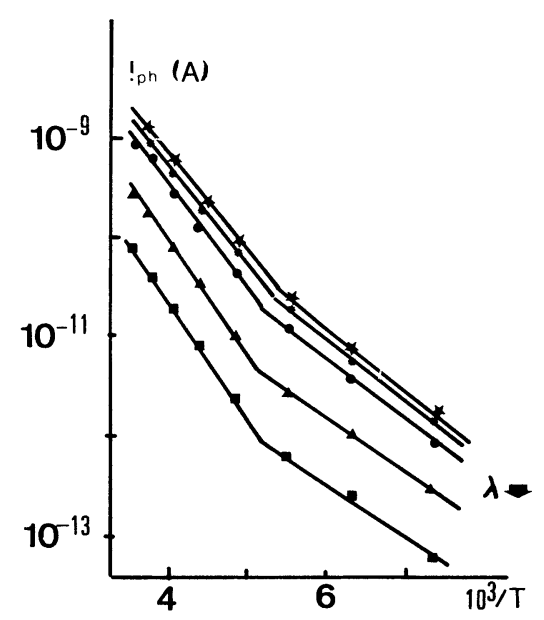

(a)

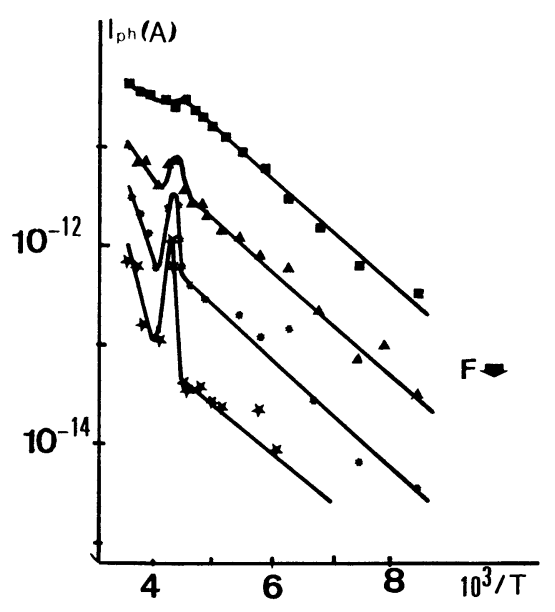

(b)

FIG. 6. - Photocourant en fonction de la température : $a$ ) forts éclairements ; b) faibles éclairements.

a) Au-dessous de $190 \mathrm{~K}$ aux forts éclairements (Fig. 6a) et de $220 \mathrm{~K}$ aux faibles éclairements (Fig. 6b) l'énergie d'activation est constante et égale à $0,12 \mathrm{eV}$, c'est-à-dire que dans ce domaine de température la 
photoconduction est contrôlée par l'énergie d'activation de saut dans les états qui gouvernent la mobilité des trous [12,13], comme 1'a montré Spear pour le silicium [14].

B) Aux forts éclairements et pour des températures supérieures à $190 \mathrm{~K}$, l'énergie d'activation varie avec la longueur d'onde du rayonnement (Fig. 7). Cette variation a été expliquée par Davis et Knights [15], en introduisant un temps de thermalisation, temps nécessaire à une paire électron-trou pour perdre son excès d'énergie cinétique par émission de phonons à la fréquence $v_{\mathrm{p}}$. Si $r$ est la distance électron-trou à l'instant $t$ et $E_{\mathrm{g}}$ la largeur de la bande interdite, on a

$$
t=\frac{\left(h v-E_{\mathrm{g}}\right)+\frac{e^{2}}{4 \pi \varepsilon r}}{h v_{\mathrm{p}}} .
$$

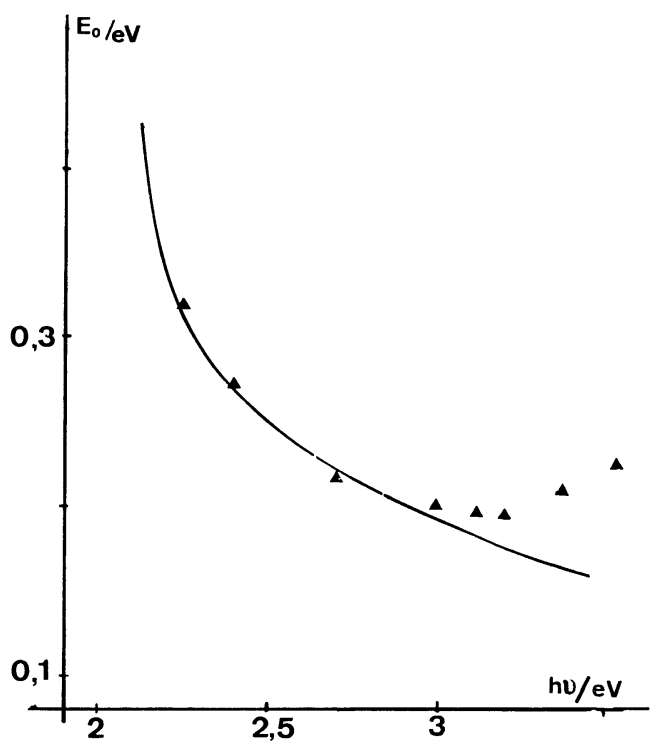

FIG. 7. - Variation de l'énergie d'activation avec l'énergie du rayonnement. Courbe théorique (éq. (4)) en traits pleins.

La distance $r_{0}$ entre l'électron et le trou au moment de la séparation est, en présence d'un champ électrique $E$, solution de l'équation.

$$
\frac{h v_{\mathrm{p}}^{2}}{D} r_{0}^{2}=\left(h v-E_{\mathrm{g}}\right)+\frac{e^{2}}{4 \pi \varepsilon r_{0}}+e E r_{0}
$$

dans laquelle $D$ est le coefficient de diffusion. L'énergie d'activation est alors donnée par

$$
E_{0}=\frac{e^{2}}{4 \pi \varepsilon r_{0}}+e E r_{0} \text {. }
$$

A partir des équations (3) et (4), nous avons recherché le meilleur accord entre les valeurs théoriques et expérimentales. Comme il n'est pas possible de déterminer séparément $D$ et $v_{\mathrm{p}}$ nous avons fixé

$$
D=0,15 \mathrm{~m}^{2} \mathrm{~s}^{-1} \quad[16] \text {. }
$$

Le meilleur accord (courbe en traits pleins) a été obtenu pour $E_{\mathrm{g}}=2,238 \mathrm{eV}$ et $v_{\mathrm{p}}=5 \times 10^{13} \mathrm{~Hz}$. Des calculs identiques menés sur les résultats obtenus par Pai nous ont conduit à des valeurs voisines [17].

$\gamma$ ) Dans le domaine des faibles éclairements et pour des températures supérieures à $220 \mathrm{~K}$, le phénomène est extrêmement complexe; les énergies d'activation varient avec le domaine de température et l'intensité lumineuse (Fig. 8) ainsi qu'avec la longueur d'onde. Ces variations ne peuvent être entièrement expliquées par les théories récentes $[18,19]$, car dans le sélénium le photocourant est toujours supérieur au courant d'obscurité.

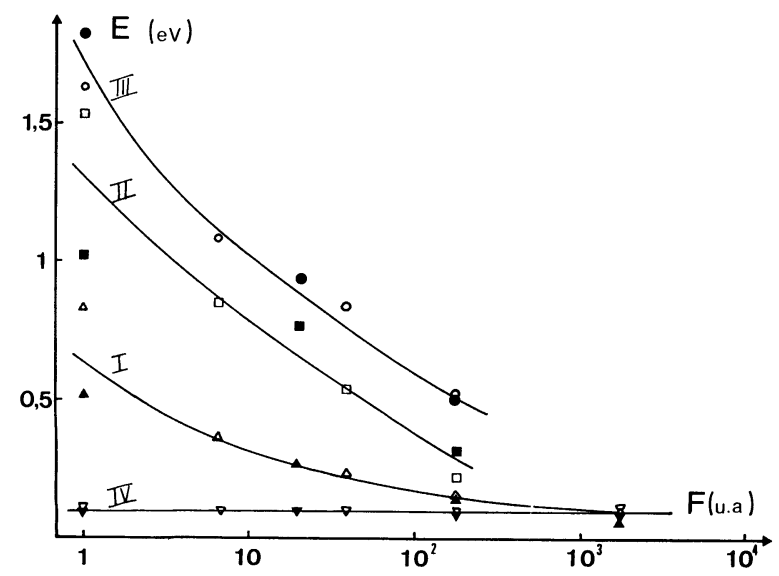

FIG. 8. - Variation de l'énergie d'activation avec l'intensité lumineuse. Région I. $T>256 \mathrm{~K}$; II. $256 \mathrm{~K}>T>238 \mathrm{~K}$; III. $238 \mathrm{~K}>T>222 \mathrm{~K}$; IV. $T<222 \mathrm{~K}$.

6. Conclusion. - Bien que la photoconduction soit un phénomène très complexe, il nous a été possible de déterminer à partir d'une étude systématique :

- que les couches étaient composées en majeure partie d'anneaux $\mathrm{Se}_{8}$,

- qu'aux basses températures la photoconduction est contrôlée par les états qui gouvernent la mobilité, alors qu'à la température ambiante elle est assistée par le champ électrique suivant une loi de Poole-Frenkel, - la largeur de bande interdite, $E_{\mathrm{g}}=2,238 \mathrm{eV}$, valeur en bon accord avec celles déterminées par d'autres méthodes.

\section{Bibliographie}

[1] Abdulayev, G. B., Asadou, Y. G., Mamedou, K. P., The physics of selenium and tellurium, W. C. Cooper Ed. (Pergamon Press, New York), 1969 p. 179.

[2] Juskas, G., Vengris, S. et Visckas, J., Proc. 5th Int. Conf. on amorphous and liquid semicond. (Garmisch-Partenkirschen) 1973363.
[3] Mimomura, S., Aoki, K., Shimomura, O. et Tanaka, K., Proc. 6th Int. Conf. on amorphous and liquid semicond., Elect. Phenom., Leningrad (1975) 289.

[4] Lucovsky, G., The physics of selenium and tellurium, W. C. Cooper Ed. (Pergamon Press, New York) 1969 p. 253. 
[5] Lucovsky, G., Proc. 5th Int. Conf. on amorphous and liquid semicond. (Garmisch-Partenkirschen), 1973, 1099.

[6] Zirke, J. et TAusend, A., Proc. 6th Int. Conf. on amorphous and liquid semicond., Structure and prop. (Leningrad 1975) 310.

[7] Cooper, W. C. et Westbury, R. A., Selenium, R. A., ZINGaro et CoOPER W. C., Eds. (Van Nostrand, New York), 1974 p. 87.

[8] Vautier, C. et Viger, C., J. Non-Cryst. Solids, (à paraître).

[9] Kaplow, R. A., Rowe, T. A. et Averbach, B. L., Phys. Rev. 168 (1968) 1068.

[10] Pai, D. et Ing, S. W., Phys. Rev. 173 (1968) 729.

[11] Siemsen, K. J. et Fenton, E. W., Phys. Rev. 161 (1967) 632.
[12] Hartke, J. L., Phys. Rev. 125 (1962) 1177.

[13] Tabak, M. D. et Warter, P. J., Phys. Rev. 173 (1968) 899. [14] Spear, W. E., Loveland, R. J. et Sharbaty, A. Al., J. Non-Cryst. Solids 15 (1974) 410.

[15] Knights, J. C. et Davis, E. A., J. Phys. Chem. Solids 35 (1974) 543.

[16] Davis, E. A., J. Non-Crystall. Solids 4 (1970) 107.

[17] Viger, C. et Vautier, C., Phys. of Non-Crystall. Solids (Clausthal-Zellerfeld) 1976.

[18] Simmons, J. G. et Taylor, G. W., J. Phys. C 7 (1974) 3051.

[19] Mott, N. F., Davis, E. A. et Street, R. A., Phil. Mag. 32 (1975) 961. 